\title{
A Distributed Filter Within a Switching Converter for Application to 3-D Integrated Circuits
}

\author{
Jonathan Rosenfeld and Eby G. Friedman, Fellow, IEEE
}

\begin{abstract}
A design methodology for distributing a buck converter filter for application to 3-D circuits is described. The 3-D filter exploits transmission line properties, permitting the generation and distribution of power supplies to different planes. As compared to a conventional $L C$ filter, the proposed filter only requires on-chip capacitors without the use of on-chip inductors. Additionally, the physical structure of the filter simultaneously enables the distribution of the current to the load while filtering the switching signal at the input. A case study in a 0.18- $\mu \mathrm{m}$ CMOS 3-D technology demonstrates the generation of a $1.2 \mathrm{~V}$ power supply delivering $700 \mathrm{~mA}$ peak current.
\end{abstract}

Index Terms-3-D integrated circuits, dc-dc buck converters, distributed filters.

\section{INTRODUCTION}

I N THE ERA of rapid technology scaling, the performance and reliability of integrated circuits (IC) have reached limits that are difficult to surpass. As a result, novel design methodologies for high performance, high complexity ICs are required. 3-D nanoscale technology can provide the required characteristics of future state-of-the-art integrated systems. With 3-D circuits [1], new design challenges arise. One primary requirement of 3-D integrated systems is diverse, high quality, and reliable power [2]. This fundamental issue of power generation and distribution in 3-D circuits is explored in this paper.

3-D integrated circuits are comprised of multiple planes with many circuit domains. The different planes are typically dedicated to a specific function, forming a highly heterogeneous system [1], [3], [4]. As an example, RF, analog, communications, and digital circuits are typically located on different planes, requiring several power supply voltages, as illustrated in Fig. 1. In this example, $V_{\mathrm{dd} 1}, V_{\mathrm{dd} 2}, V_{\mathrm{dd} 3}$, and $V_{\mathrm{dd} 4}$ are generated from the primary power supply $V_{\mathrm{dd}}$.

Multiple circuit domains require several power supplies to reliably operate and provide sufficient and stable current. To provide circuit domains with the appropriate power supplies, dc-dc converters are distributed across each plane [5] as it is often impractical to provide external power supplies due to the

Manuscript received April 21, 2009; revised October 07, 2009. First published April 26, 2010; current version published May 25, 2011. This work was supported in part by the National Science Foundation under Contract CCF0541206, CCF-0811317, and CCF-0829915, grants from the New York State Office of Science, Technology \& Academic Research to the Center for Advanced Technology in Electronic Imaging Systems, and by grants from Intel Corporation, Eastman Kodak Company, and Freescale Semiconductor Corporation.

J. Rosenfeld and E. G. Friedman are with the Department of Electrical and Computer Engineering, University of Rochester, Rochester, NY 14627 USA (e-mail: rosenfeld.jonathan@gmail.com).

Digital Object Identifier 10.1109/TVLSI.2010.2045601

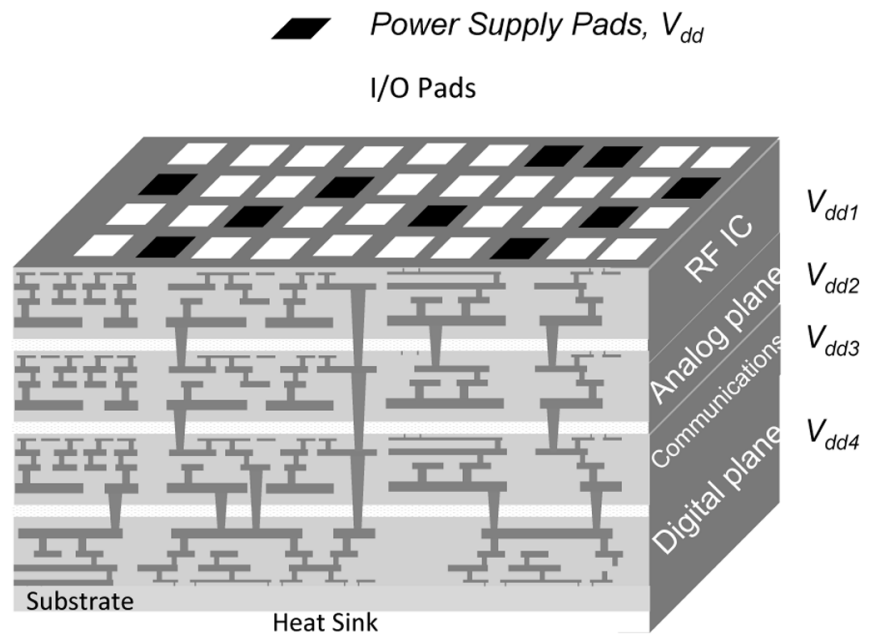

Fig. 1. 3-D circuit with multiple power supplies.

limited number of input pins. Moreover, planes located far from the faces of the 3-D cube require a large number of expensive 3-D vias to distribute the power supply across the plane.

To alleviate these difficulties, dc-dc converters are distributed on-chip, generating a specific voltage required by the different circuit blocks within each plane of a 3-D system. A suitable dc-dc converter for low power applications is a buck converter [5], [6]. A buck converter generates an output supply voltage smaller than the input supply voltage [5].

The filter portion of a buck converter to generate and distribute power supplies in 3-D integrated circuits is the primary focus of this paper. The proposed filter is comprised of on-chip interconnects and capacitors, eliminating the need for an on-chip inductor [7]. For a specific dc voltage ripple, the distributed filter produces the target transfer function, passing the dc component of the input signal while attenuating the high frequency harmonics.

This paper is organized into five sections. Background on the operation of a conventional buck converter is reviewed in Section II. In Section III, a methodology for designing these circuits is described. To exemplify the proposed approach, a case study is described in Section IV, while a performance analysis of the distributed filter is presented in Section V. Some conclusions are offered in Section VI.

\section{BACKGROUND}

A standard topology of a buck converter for high performance microprocessors is depicted in Fig. 2(a) [5]. The power MOSFETs produce an ac signal at node A by a signal controlled by a pulse width modulator (PWM) [8], [9], as shown in Fig. 2(b). 


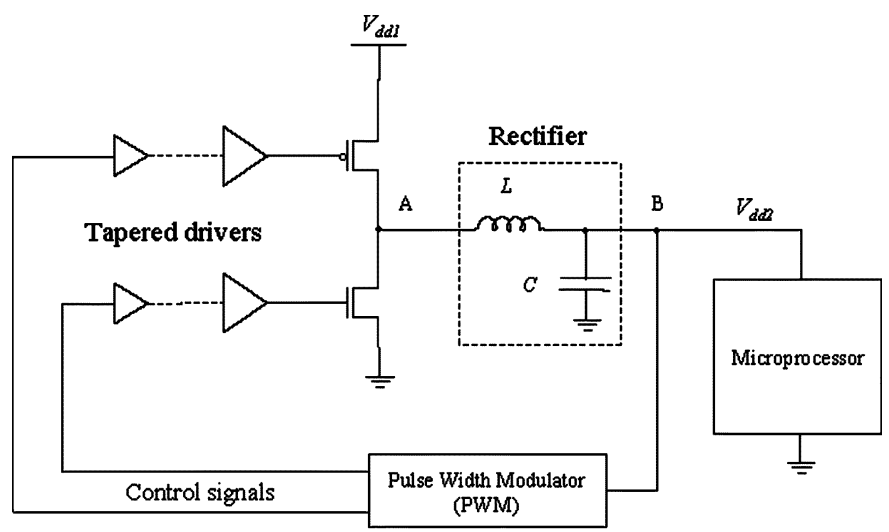

(a)

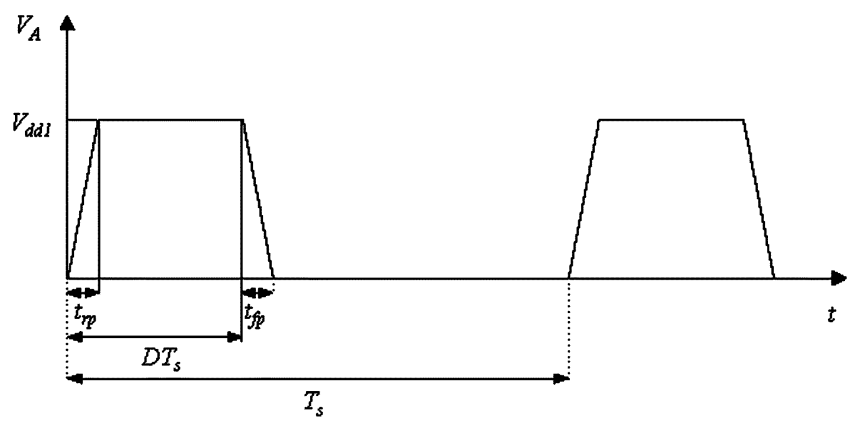

(b)

Fig. 2. Conventional dc-dc converter: (a) buck converter circuit [5] and (b) signal at the output of the power MOSFETs (node A).

The ac signal at node A is filtered by a filter composed of a second-order low pass band $L C$ filter. Assuming the resonance frequency $1 / 2 \pi \sqrt{L C}$ is less than the switching frequency of the power MOSFETs, the filter only passes the dc component of the signal and a residue composed of the high frequency harmonics. The dc component of the signal shown in Fig. 2(b) is

$$
\frac{1}{T_{s}} \int_{0}^{T_{s}} V_{A} d t=\frac{V_{\mathrm{dd} 1}\left(2 D T_{s}-t_{r p}+t_{f p}\right)}{2 T_{s}}
$$

where $D, T_{s}$, and $V_{\mathrm{dd} 1}$ are the duty cycle, time period, and input power supply, respectively, and $t_{r p}$ and $t_{f p}$ are the rise and fall times, respectively, of the switching signal produced by the PWM circuit. In the case of $t_{r p}=t_{f p}$, (1) reduces to

$$
V_{\mathrm{dd} 2}=V_{D C \mid t_{r p}=t_{f p}}=D V_{\mathrm{dd} 1} \text {. }
$$

Hence, the buck converter produces an output voltage $V_{\mathrm{dd} 2}$ at node B equal to $D V_{\mathrm{dd} 1}$.

The power transistors are typically large in physical size and are therefore driven by tapered buffers [10]. These buffers are controlled by the PWM circuit. The feedback PWM circuit senses the output voltage supply $V_{\mathrm{dd} 2}$ at node B and modifies the control signal to ensure that the appropriate duty cycle $D$ is produced at node A. In this manner, the output voltage is maintained at the desired value while compensating for variations in the load current and input voltage. The performance and functionality of the different circuit domains under load current and dc input voltage variations are dependent upon the maximum voltage fluctuations generated by the buck converter, which is therefore regulated to provide the target voltage [5].
The performance of a buck converter can be improved by integrating these converters on-chip. In this manner, the parasitic losses associated with the interconnects among the nonintegrated components of the dc-dc converter are significantly decreased. Moreover, integrated converters benefit by advances in on-chip technologies and high operating frequencies. Monolithic fully integrated dc-dc converters can therefore achieve higher efficiency as compared to nonintegrated converters [11]-[14].

Integrating dc-dc converters on-chip in both 2-D and 3-D technologies, however, imposes challenges as the on-chip integration of large inductive and capacitive elements is problematic. A significant issue is the poor parasitic impedance characteristics exhibited by the on-chip inductors [15], [16], which degrades the performance of the on-chip converter. To improve the quality factor of a $2 \mathrm{nH}$ inductor and reduce the ripple current within the inductors, magnetic coupling between two on-chip inductors has been used in a dc-dc converter [17]. Although the size and magnitude of the on-chip inductors and capacitors required to implement a buck converter are reduced with increasing switching frequency, the on-chip passive devices comprising the filter are large and cannot be practically integrated in the megahertz frequency regime [18]-[20].

A 3-D technology provides several advantages as compared to a 2-D technology. When the on-chip capacitors used by a distributed filter are implemented with active devices, less metallization resources are required to connect these capacitors to different sections of the filter as compared to a 2-D technology. Since in 3-D circuits each plane has a dedicated active device layer, routing congestion is reduced due to the smaller distance between the interconnect and the corresponding capacitor. Also, in a 2-D technology, the input power supply to the power MOSFETs (which are located within the substrate) is connected by the lowest metal layers, therefore, additional routing resources are required.

In a 3-D technology, the plane closest to the input power supply accommodates the power MOSFETs, saving metallization resources. The capacitance and inductance of the through silicon vias are exploited, reducing the required length of the interconnects and the size of the capacitors. An inherent benefit of utilizing the proposed filter in 3-D systems is that the generation and distribution of the power supply occur simultaneously, while in a 2-D technology, the filter is only used to generate the power supply.

To obtain insight into the operation of a distributed filter as compared to a lumped $L C$ filter, consider the transfer function of two types of filters, as depicted in Fig. 3. A second-order low pass $L C$ filter is used in a typical conventional dc-dc converter. When the effective output resistance $R_{d}$ of the power MOSFETs as well as the effective series resistance of the inductor are included, two poles at different frequencies are formed, resulting in a roll-off slope of $-20 \mathrm{~dB} /$ decade in the megahertz and -40 $\mathrm{dB} /$ decade in the gigahertz frequency range. The frequency behavior of a distributed filter in the megahertz frequency range is therefore similar to a lumped $L C$ filter, as can be observed in Fig. 3. When $R_{d}$ and the interconnect resistance are included in the analysis, a pole in the megahertz frequency is formed, resulting in a roll-off slope of $-20 \mathrm{~dB} /$ decade. Note that in this 


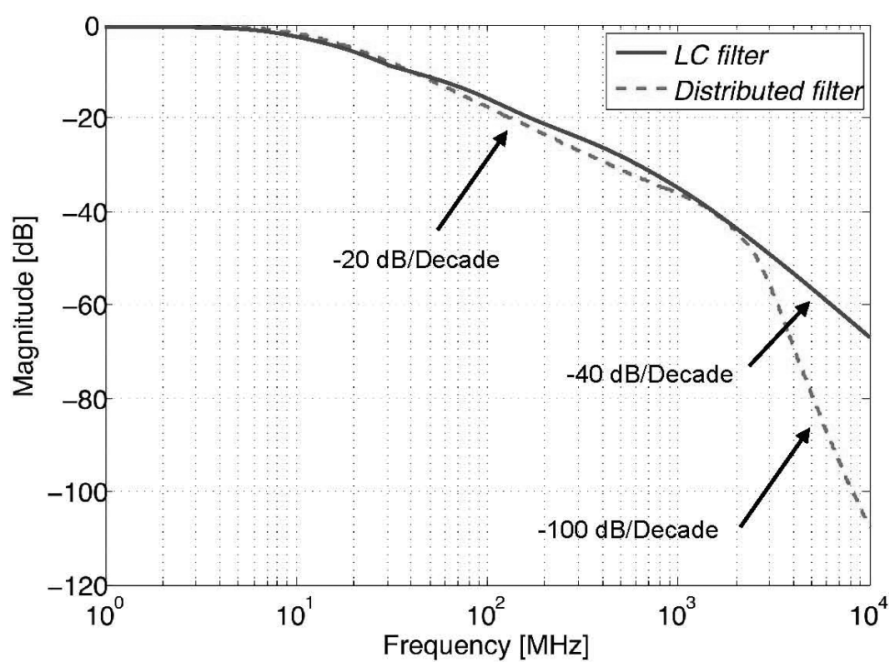

Fig. 3. Transfer functions of $L C$ and distributed filters.

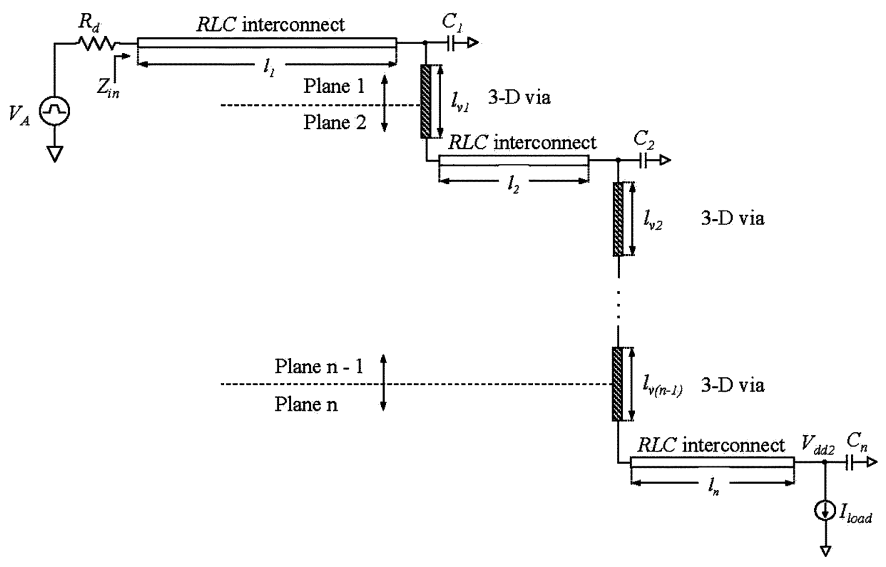

Fig. 4. Distributed filter.

example, a sharp $-100 \mathrm{~dB} / \mathrm{decade}$ roll-off slope is formed in the gigahertz frequency range, suppressing the high frequency harmonics of the ac signal produced by the power MOSFETs. The distributed nature of the proposed filter forms multiple poles at approximately the same high frequency, resulting in a large negative slope.

\section{Design Methodology}

A design methodology for a distributed buck converter is described in this section. Design guidelines are provided based on the expressions developed here. The physical structure of the distributed filter as well as the current load characteristics are described in Section III-A. In Section III-B, the transfer function of the filter is used to determine the condition that satisfies a target power supply ripple while the efficiency and area of the 3 -D filter are determined in Section III-C. Finally, design guidelines are described in Section III-D.

\section{A. Physical Structure and Current Load Properties of a 3-D Filter}

The proposed distributed filter is depicted in Fig. 4. The filter is driven by power MOSFETs [see Fig. 2(a)] which are modeled as a voltage source $V_{A}$ followed by an effective resistance $R_{d}$.

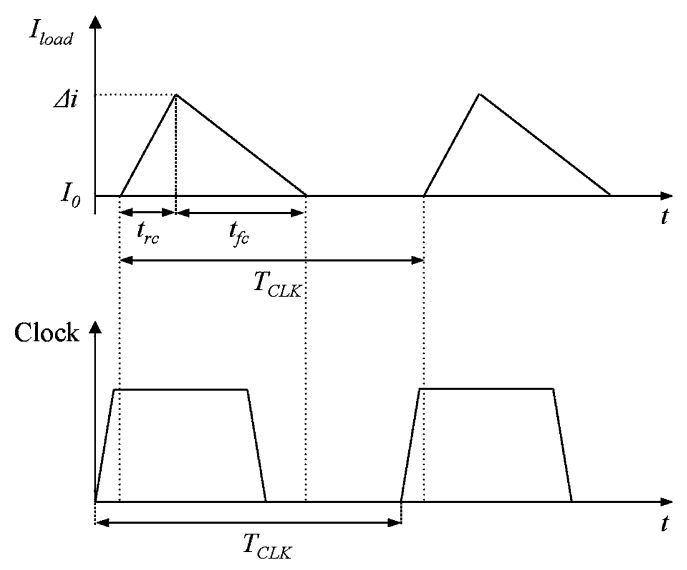

Fig. 5. Current load profile.

The voltage source $V_{A}$ is assumed to be periodic, as illustrated in Fig. 2(b).

The filter is composed of transmission lines terminated with lumped capacitances. The inter-plane structure is connected by 3 -D vias. At the target plane $n$, the load is represented by a periodic current load and a reference clock signal, as shown in Fig. 5. Note that the current load characterizes the approximate current profile of a specific circuit module on a plane. $I_{\text {load }}$ remains at $I_{0}$ during clock low, providing dc current flow by the power supply. As with a conventional buck converter, a feedback PWM circuit senses the output node of the filter and adjusts the duty cycle of the signal driving the power MOSFETs [see Fig. 2(a)].

In 3-D circuits, the ability to deliver current is primarily limited by the $3-D$ vias. The maximum current that can be delivered through a single 3-D via therefore determines the current magnitude

$$
I_{0}+\Delta i=J_{\mathrm{via}, \max } \cdot A_{\mathrm{via}} \cdot V
$$

where $J_{\text {via,max }}, A_{\text {via }}$, and $V$ are, respectively, the maximum current density, cross-sectional area, and number of 3-D vias on the same plane. Consequently, the maximum cross-sectional area of the interconnects (see Fig. 4) distributing the current within the different planes is

$$
A_{\text {int }}=\frac{I_{0}+\Delta i}{J_{\text {int }, \max }}=\left(\frac{J_{\text {via, } \max }}{J_{\text {int }, \text { max }}}\right) \cdot A_{\text {via }} \cdot V
$$

where $J_{\text {int,max }}$ is the maximum current density of the interconnect.

In practical circuits, however, a significant amount of current is sunk by the load. To satisfy this requirement, multiple structures $N$, as depicted in Fig. 4, are connected in parallel, delivering $N\left(I_{0}+\Delta i\right)$ amperes. In this case, the number of 3-D vias within the filter on each plane is equal to the number of parallel connected structures, $V=N$. The effective resistance and inductance per unit length of the interconnects and 3-D vias, as well as the output resistance of the driver (see Fig. 4), are $N$ times smaller. The capacitance per unit length of the interconnects and 3-D vias, as well as the on-chip lumped capacitors, are $N$ times larger. 


\section{B. Transfer Function of a 3-D Filter}

To characterize the impedance of the filter (between $R_{d}$ and $\left.I_{\text {load}}\right)$, the overall transfer function is determined based on the $A B C D$ matrices. Hence, the overall $A B C D$ matrix of a filter spanning $n$ planes is

$$
\left[\begin{array}{cc}
\tilde{A} & \tilde{B} \\
\tilde{C} & \tilde{D}
\end{array}\right]=\left[\begin{array}{cc}
1 & R_{d} \\
0 & 1
\end{array}\right] \cdot\left[\begin{array}{ll}
A^{\prime} & B^{\prime} \\
C^{\prime} & D^{\prime}
\end{array}\right]
$$

The right matrix in the right-hand side of (5) is

$$
\begin{array}{r}
{\left[\begin{array}{ll}
A^{\prime} & B^{\prime} \\
C^{\prime} & D^{\prime}
\end{array}\right]=\prod_{i=1}^{n}\left(\left[\begin{array}{cc}
\cosh \gamma l_{i} & Z_{0} \sinh \gamma l_{i} \\
\frac{\sinh \gamma l_{i}}{Z_{0}} & \cosh \gamma l_{i}
\end{array}\right]\left[\begin{array}{cc}
1 & 0 \\
j \omega C_{i} & 1
\end{array}\right]\right.} \\
\left.\cdot\left[\begin{array}{cc}
\cosh \gamma_{v} l_{v i} & Z_{v 0} \sinh \gamma_{v} l_{v i} \\
\frac{\sinh \gamma_{v} l_{v i}}{Z_{v 0}} & \cosh \gamma_{v} l_{v i}
\end{array}\right]\right)
\end{array}
$$

where $Z_{0}$ and $\gamma$ are the characteristic impedance and propagation constant of the $R L C$ interconnects, respectively, $l_{i}$ and $C_{i}$ are the interconnect length and capacitance on the $i$ th plane, respectively, $Z_{v 0}$ and $\gamma_{v}$ are the characteristic impedance and propagation constant of the $3-\mathrm{D}$ vias, respectively, and $l_{v i}$ is the length of the $3-\mathrm{D}$ via on the $i$ th plane. The transfer function of the filter is

$$
H_{\text {rect }}(j \omega)=\frac{1}{\tilde{A}}
$$

where $\tilde{A}$ and $\tilde{B}$ are obtained from (5), and $\omega_{\mathrm{CLK}}$ and $\omega_{s}$ are the radian frequency of the clock and switching signals, respectively.

Since a practical filter within a buck converter does not provide ideal low pass characteristics, the signal at node B, shown in Fig. 2(a), carries a small amount of high frequency harmonics generated by the switching power MOSFETs. Hence, the voltage at node $B$ is

$$
V_{\mathrm{dd} 2}(t)=V_{D C}+V_{\text {ripple }}(t)
$$

where $V_{D C}$ is the dc component of the output voltage described by (1) and $V_{\text {ripple }}(t)$ is the voltage ripple transferred by the nonideal characteristics of the filter. When only the fundamental harmonic is passed, $V_{\text {ripple }}(t)$ exhibits a sinusoidal behavior

$$
V_{\text {ripple }}(t)=V_{r} \sin \left(\omega_{s} t\right)
$$

To satisfy a target ripple voltage $V_{r}$ (peak-to-peak), the magnitude of the filter transfer function at the switching frequency $f_{s}$ has to be below a specific value. To satisfy this objective, consider the output signal in the frequency domain

$$
\left|V_{\mathrm{dd} 2}(s)\right|=\left|H_{\mathrm{rect}}(s)\right| \cdot\left|V_{A}(s)\right| \text {. }
$$

The periodic input signal $V_{A}$ can be represented by a Fourier series

$$
v_{A}(t)=\sum_{k=-\infty}^{\infty} a_{k} e^{j k \omega_{s} t}
$$

where $a_{k}$ is the $k$ th harmonic of the signal. In the case of the signal illustrated in Fig. 2(b) and assuming $t_{r p}=t_{f p}=t_{r}$, the fundamental harmonic (positive and negative) is

$a_{ \pm 1}=\left(\frac{V_{d d 1} T_{s}}{4 t_{r} \pi^{2}}\right) \cdot\left(e^{\mp j \omega_{s} t_{r}}\left(1-e^{\mp j 2 \pi D}\right)+e^{\mp j 2 \pi D}-1\right)$.

Equation (10) implies that the required amplitude of the transfer function for a specific ripple voltage $V_{r}$ is

$$
\left|H_{\text {rect }}\left(j \omega_{s}\right)\right| \leq \frac{\frac{V_{r}}{2}}{2\left|a_{1}\right|}=\frac{V_{r}}{4\left|a_{1}\right|} .
$$

Once the current profile of a circuit is determined, the interconnect length $l_{1}$ to $l_{n}$, shown in Fig. 4, and the required ripple voltage $V_{r}$ are chosen. Based on (7), the magnitude of the transfer function at $\omega_{s}$ is plotted as a function of the capacitances $C_{1}$ to $C_{n}$. The interconnect length and capacitance are chosen to satisfy (13). Since these design expressions are complex and unsuitable for manual calculations, numerical techniques are used.

An important issue is the duty cycle of the signal driving the power MOSFETs (see Fig. 4) that produces the correct power supply voltage. Note that in this case, the duty cycle determined from (2) does not provide the proper dc voltage level. This behavior occurs since the signal at the input of a distributed filter is degraded by the resistance $R_{d}$ and the input impedance of the filter $Z_{\text {in }}$, forming a voltage divider. To obtain the duty cycle required for a specific dc voltage, consider the input impedance of the filter at DC

$$
Z_{\text {in }}(0)=\frac{V_{D C} A^{\prime}(0)+I_{D C} B^{\prime}(0)}{V_{D C} C^{\prime}(0)+I_{D C} D^{\prime}(0)}
$$

where $A^{\prime}, B^{\prime}, C^{\prime}$, and $D^{\prime}$ are defined in (6), and $I_{D C}$ is the dc component of the current load

$$
I_{D C}=I_{0}+\frac{\Delta i}{2 T_{\mathrm{CLK}}} \cdot\left(t_{r c}+t_{f c}\right) .
$$

The dc component of the signal at the input of the filter (including $R_{d}$ in Fig. 4) is

$$
D V_{\mathrm{dd} 1}=D_{\mathrm{PWM}} V_{\mathrm{dd} 1}\left|\frac{Z_{\mathrm{in}}(0)}{Z_{\mathrm{in}}(0)+R_{d}}\right|
$$

the dc voltage transferred by the filter to the target plane. In (16), $D_{\mathrm{PWM}}$ is the duty cycle provided by the PWM feedback circuit [see Fig. 2(a)]. Consequently, to achieve a specific dc voltage at the output of the filter, the duty cycle in (2) is

$$
D_{\mathrm{PWM}}=D \cdot\left|1+\frac{R_{d}}{Z_{\mathrm{in}}(0)}\right| .
$$

Observe from (17) that $D_{\mathrm{PWM}}$ is always larger than the original duty cycle $D$ obtained from (2), limiting the magnitude of the generated power supply. When the interconnects within the distributed filter are resistive, $D_{\mathrm{PWM}}$ approaches $D$ (no reflections occur at the input). It is typically preferable to design the filter to ensure that $D_{\mathrm{PWM}}$ is closer to $D$ to provide a large tuning range for the PWM circuit. 


\section{Efficiency and Area of a 3-D Filter}

An important property of a buck converter is the power efficiency. The efficiency of a distributed filter within a buck converter is

$$
\eta_{\text {rect }}=\frac{P_{\text {load }}}{P_{\text {load }}+P_{\text {rect }}} \times 100 \%
$$

where $P_{\text {load }}$ is the average power delivered to the load and $P_{\text {rect }}$ is the average power consumed by the filter. The power expressions $P_{\text {load }}$ and $P_{\text {rect }}$ are, respectively,

$$
\begin{aligned}
& P_{\text {load }}=V_{\mathrm{dd} 2} I_{D C} \\
& P_{\text {rect }}=V_{A, \text { rms }}^{2}\left|\frac{Z_{\text {in }}\left(j \omega_{s}\right)}{Z_{\text {in }}\left(j \omega_{s}\right)+R_{d}}\right|^{2} \cdot \Re\left\{\frac{1}{Z_{\text {in }}\left(j \omega_{s}\right)}\right\}
\end{aligned}
$$

where $V_{A, \mathrm{rms}}$ is the root-mean-square value of the driving signal with a duty cycle $D_{\mathrm{PWM}}$, and $Z_{\mathrm{in}}\left(j \omega_{s}\right)$ are, respectively

$$
\begin{aligned}
V_{A, \mathrm{rms}} & =\sqrt{\frac{1}{T_{s}} \int_{t_{0}}^{t_{0}+T_{s}}\left|v_{A}(t)\right|^{2} d t} \\
Z_{\text {in }}\left(j \omega_{s}\right) & =\frac{V_{D C} A^{\prime}\left(j \omega_{s}\right)+I_{D C} B^{\prime}\left(j \omega_{s}\right)}{V_{D C} C^{\prime}\left(j \omega_{s}\right)+I_{D C} D^{\prime}\left(j \omega_{s}\right)} .
\end{aligned}
$$

The area occupied by a 3-D filter is

$$
\begin{aligned}
A_{3-D, \text { rect }}= & \left(\sum_{i=1}^{n}\left(W_{\text {metal }, i} \cdot l_{i}+A_{C_{i}}\right)+\sum_{i=1}^{n-1} A_{\mathrm{via}, i}\right) \\
& \cdot N+S_{i} \cdot l_{i} \cdot n \cdot(N-1)
\end{aligned}
$$

where $A_{C_{i}}$ and $A_{\mathrm{via}, i}$ are the area occupied by the on-chip capacitors and 3-D vias on the $i$ th plane, respectively. $W_{\text {metal }, i}$ and $S_{i}$ are the width and spacing of the interconnects on the $i$ th plane, respectively. As mentioned in Section III-A, $n$ and $N$ are, respectively, the number of planes spanned by the filter and the number of parallel connected filter structures. The generation and distribution of additional power supplies, as illustrated in Fig. 1, requires the use of additional dc-dc converters and distributed filters.

\section{Design Guidelines}

In order to provide a comprehensive perspective of the design space, several design parameters that affect the performance of the distributed filter are investigated. To determine the required magnitude of the transfer function of the filter for different output voltage ripples and dc voltages, consider Fig. 6. The output dc voltage ranges between 0.8 and $3 \mathrm{~V}$ (assuming a $3.3 \mathrm{~V}$ input dc voltage), while the required voltage ripple ranges between $1 \%$ and $10 \%$. As expected, the magnitude of the transfer function increases for higher voltage ripple, since a larger amplitude of the dominant harmonic of the input signal is permitted to pass. Furthermore, as the output dc voltage becomes larger, an increase in the transfer function is evident in (10). Consequently, a lower capacitance and fewer resistive interconnects are required. Once the required output dc voltage is chosen for a specific voltage ripple, permitting the target magnitude of the transfer function to be determined, the design space of the distributed filter is specified.

In this example, all of the interconnect lengths are $l=1 \mathrm{~mm}$ with a voltage ripple $V_{r}=5 \%$, input dc voltage $V_{\mathrm{dd} 1}=3.3 \mathrm{~V}$,

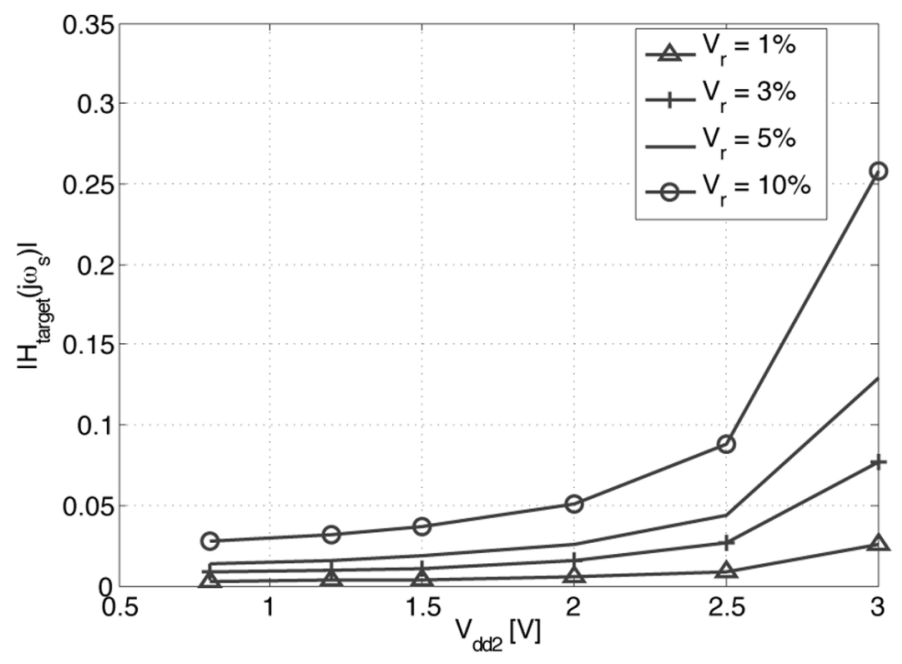

Fig. 6. Required magnitude of the transfer function.

and a switching frequency $f_{s}=100 \mathrm{MHz}$. Consider the design space of the filter, as shown in Fig. 7. The modified duty cycle $D_{\mathrm{PWM}}$, ratio between the duty cycle $D$ [see (2)] and $D_{\mathrm{PWM}}$, capacitance, and filter efficiency are depicted as a function of the output dc voltage and series resistance $R_{d}$ (see Fig. 4). The modified duty cycle $D_{\mathrm{PWM}}$ is shown in Fig. 7(a). Observations of Fig. 7(a) reveal that under the specified operating conditions, this filter cannot convert 3.3 to $3 \mathrm{~V}$ since for all values of $R_{d}$, $D_{\text {PWM }}$ is greater than one. The feasible operation of the distributed filter is therefore limited to about $2.5 \mathrm{~V}$.

To narrow the design space, recall that $D_{\mathrm{PWM}}$ is always greater than $D$ (see Section III-B). To permit a larger operational range of the feedback PWM circuit, $D_{\mathrm{PWM}}$ should be chosen close to $D$. A new design metric is therefore defined which is the ratio of $D$ to $D_{\mathrm{PWM}}$, as shown in Fig. $7(\mathrm{~b})$. This ratio should be as close to one as possible. As shown in Fig. 7(b), the ratio $D / D_{\mathrm{PWM}}$ decreases as $R_{d}$ increases. The lowest permissible ratio $D / D_{\mathrm{PWM}}$ is chosen to be 0.5 to accommodate a tradeoff between the ratio $D / D_{\mathrm{PWM}}$ and the filter efficiency.

As mentioned in the beginning of this subsection, less capacitance is required for lower conversion ratios, as shown in Fig. 7(c). As expected, with a larger series resistance of the power MOSFETs $R_{d}$, the required capacitance can be decreased to satisfy the target magnitude of the transfer function. Finally, the filter efficiency $\eta_{\text {rect }}$ is shown in Fig. 7(d). The general trend for all output DC voltages is that the filter efficiency increases with higher $R_{d}$, as evident from (18) and (20). From Figs. 7(b)-(d), the permissible range for $R_{d}$ is

$$
\begin{aligned}
R_{d}\left(\min \left(\eta_{\text {rect }}\right)\right) & <R_{d}<R_{d}\left(\frac{D_{\mathrm{PWM}}}{D}=\frac{1}{2}\right) \\
R_{d}(\max (C)) & <R_{d}<R_{d}\left(\frac{D_{\mathrm{PWM}}}{D}=\frac{1}{2}\right) .
\end{aligned}
$$

\section{CASe Study}

To demonstrate the design methodology described in Section III, an example DC-DC converter based on the MIT Lincoln Lab (MITLL) $180 \mathrm{~nm}$ 3-D integration process [21], 


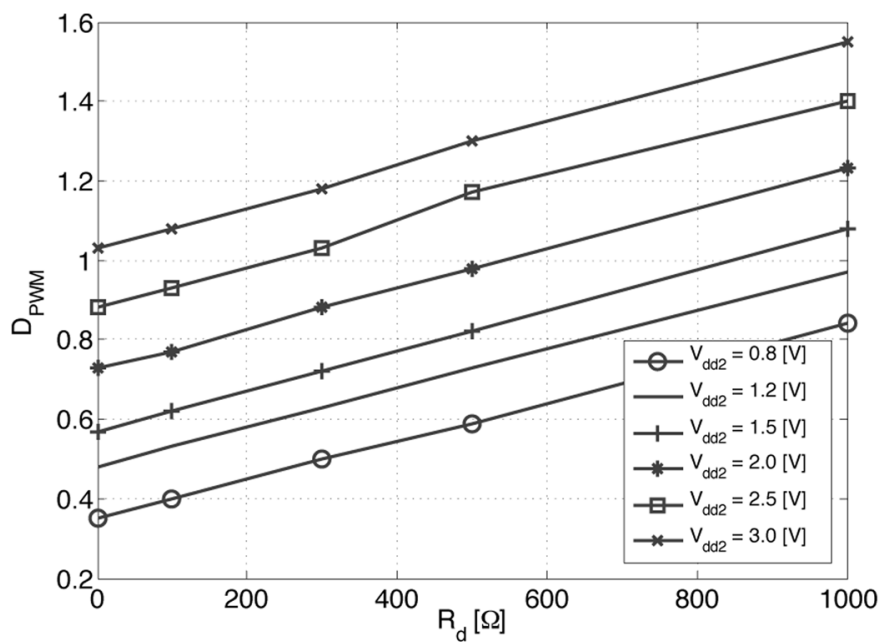

(a)

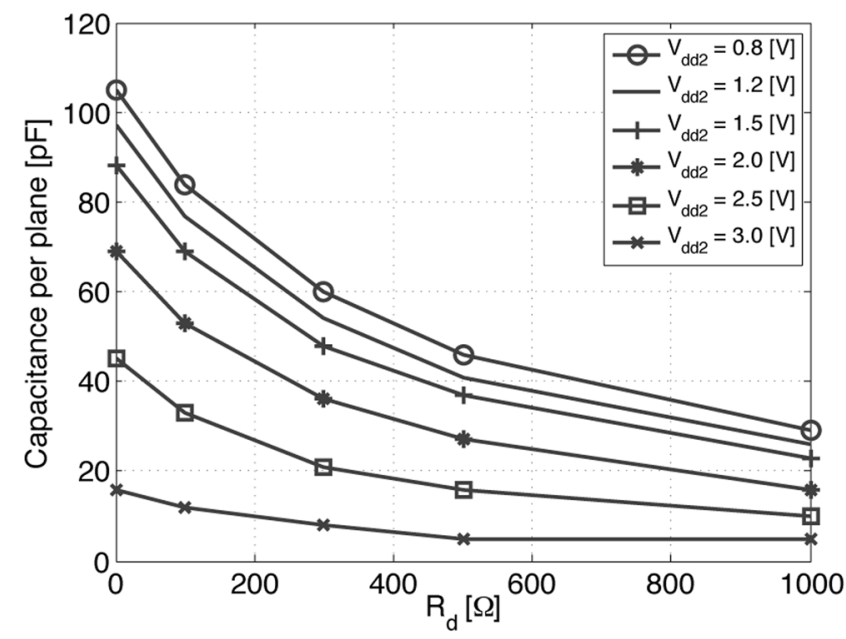

(c)

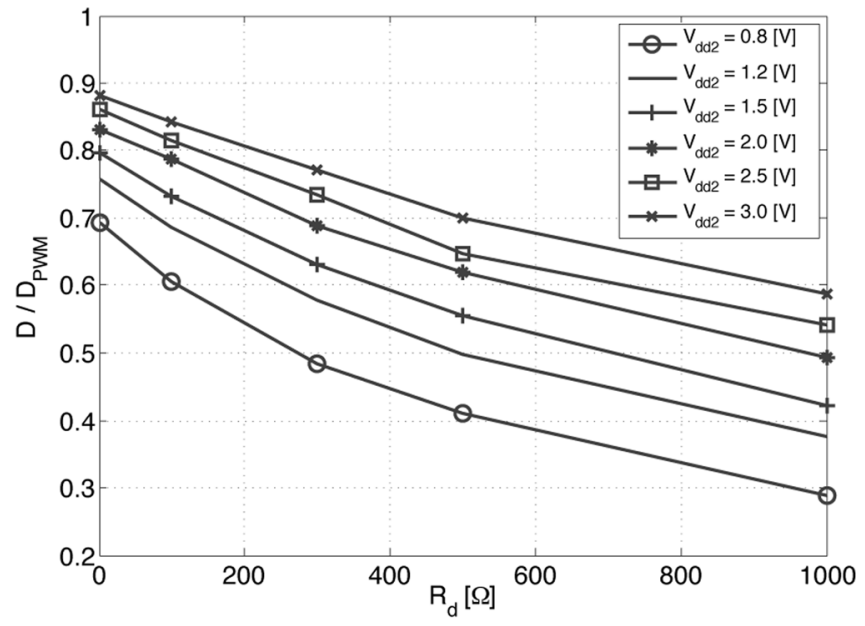

(b)

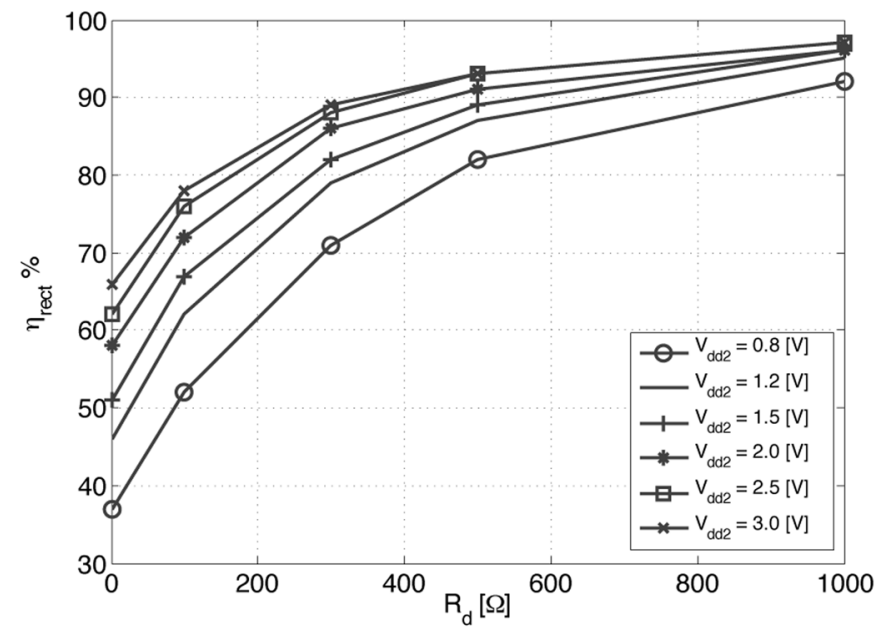

(d)

Fig. 7. Design space of a distributed filter: (a) modified duty cycle as a function of $R_{d}$; (b) duty cycle ratio as a function of $R_{d}$; (c) capacitance as a function of $R_{d}$; (d) filter efficiency as a function of $R_{d}$.

[22] is described in this section. Since the ability to deliver current is primarily limited by the $3-\mathrm{D}$ via, characterization of the maximum current that can flow through a single via is described in Section IV-A. A distributed filter based on a 3-D technology is described in Section IV-B and compared to an implementation in a 2-D technology in Section IV-C. In order to quantify the advantages of the proposed distributed filter as compared to a conventional buck converter filter, a traditional filter is described in Section IV-D for the same performance requirements as a distributed filter. SPICE simulations are performed based on the 3-D MITLL technology.

\section{A. Current Load Characterization}

The MITLL technology is a $0.18 \mu \mathrm{m}$ low power, fully depleted silicon-on-insulator (FDSOI) CMOS process where three independent wafers are physically bonded to form a 3-D integrated structure. Each plane has three aluminum metallization layers. In this technology, the maximum current density is [22]

$$
J_{A l, 3-D}=\frac{3 \mathrm{~mA}}{\mu \mathrm{m}^{2}} .
$$

Since the cross-sectional area of a 3-D via in this technology is $1.5 \times 1.5 \mu \mathrm{m}^{2}$, the maximum current that can flow through a single 3-D via, based on (3), is

$$
I_{0}+\Delta i \approx 7 \mathrm{~mA} \text {. }
$$

In this case study, the current load waveform, depicted in Fig. 5, has the following characteristics: $1 / T_{\mathrm{CLK}}=3 \mathrm{GHz}$, and $t_{r c}$ and $t_{f c}$ are $0.3 T_{\mathrm{CLK}} / 2$ and $0.7 T_{\mathrm{CLK}} / 2$, respectively.

\section{B. Distributed 3-D Filter}

To overcome the difficulty described in Subsection IV-D, a distributed filter circuit has been developed that generates and distributes the power supply to a target plane within a 3-D structure. This filter is described in this section. The resistance, inductance, and capacitance per unit length of the interconnects (Metal 3) and 3-D vias are extracted based on the predictive technology model (PTM) [23]-[25], as listed in Table I. The width of the interconnects is determined by the maximum current density of the MITLL 3-D technology. Assuming that both the interconnect and 3-D vias support the same current density 
TABLE I

$R L C$ INTERCONNECT (ALUMINUM) AND 3-D VIA IMPEDANCES

\begin{tabular}{lccc}
\hline & $R[\mathrm{~m} \Omega / \mu \mathrm{m}]$ & $L[\mathrm{pH} / \mu \mathrm{m}]$ & $C[\mathrm{fF} / \mu \mathrm{m}]$ \\
\hline \hline Interconnects & 14.5 & 1.3 & 0.5 \\
\hline 3-D via [24] & 20.40 & 0.55 & 0.37 \\
\hline
\end{tabular}

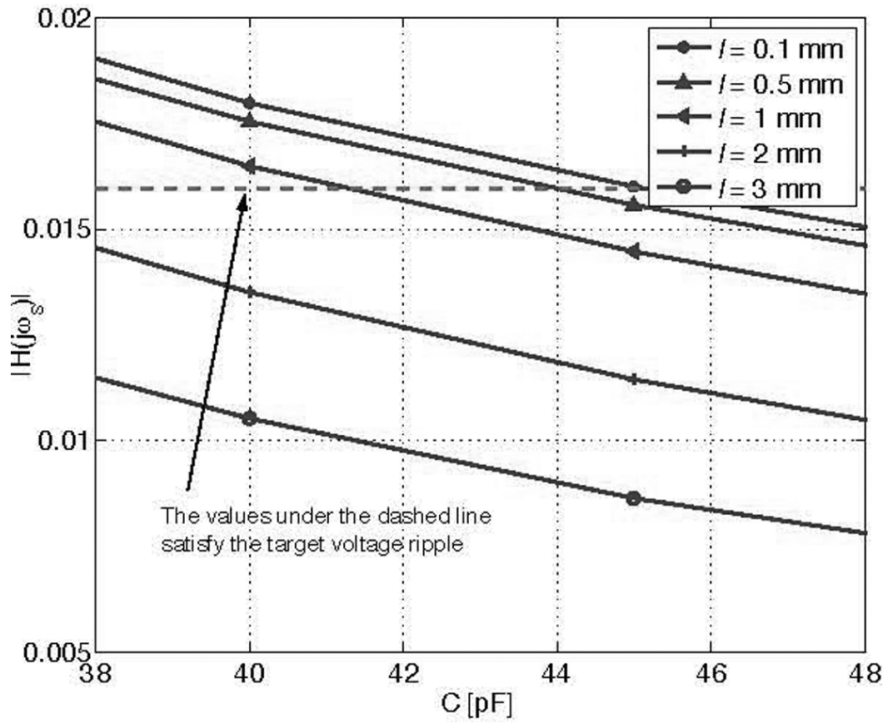

Fig. 8. Magnitude of the transfer function at $\omega_{s}$ for different line lengths and capacitances.

of $3 \mathrm{~mA} / \mu \mathrm{m}^{2}$, the maximum cross-sectional area of the interconnect determined from (4) is $1.5 \times 1.5 \mu \mathrm{m}^{2}$. The thickness of the interconnect for this technology is $630 \mathrm{~nm}$, resulting in an approximately $4 \mu \mathrm{m}$ wide line. Note that each 3 -D via is $7.34 \mu \mathrm{m}$ long, connecting three planes, as illustrated in Fig. 4.

For the same performance requirements as a conventional filter, the magnitude of the transfer function at $\omega_{s}$ for different capacitances and interconnect lengths, assuming $l=l_{1}=l_{2}=$ $l_{3}$ and $C=C_{1}=C_{2}=C_{3}$, is depicted in Fig. 8. An output resistance of the power MOSFETs $R_{d}=7.36 \Omega$ is assumed in this case study.

As the interconnect line length increases, less capacitance is required, as evident in Fig. 8. To satisfy the required voltage ripple, the target interconnect length in this example is $l=1$ $\mathrm{mm}$ with a capacitance $C=42 \mathrm{pF}$. The dc voltage at the output of the filter is shown in Fig. 9. The simulated ripple of the output dc voltage is $52 \mathrm{mV}$ ( $4.3 \%$ of the output dc voltage), satisfying the design objective of a maximum 5\% voltage ripple. In this example, the required duty cycle of the signal driving the power MOSFETs is $D_{\mathrm{PWM}}=0.73$, and the efficiency of the filter is about $88 \%$.

If additional current is required, the distributed filter can be extended by connecting multiple structures in parallel. For example, to enlarge this structure to produce $700 \mathrm{~mA}, 100$ parallel structures are required. In this case, the effective resistance and inductance per unit length, listed in Table I, and $R_{d}$ are $N$ times smaller, while the capacitance per unit length is $N$ times larger. The multiple parallel filters produce the same transfer function magnitude, as shown in Fig. 8. The same interconnect

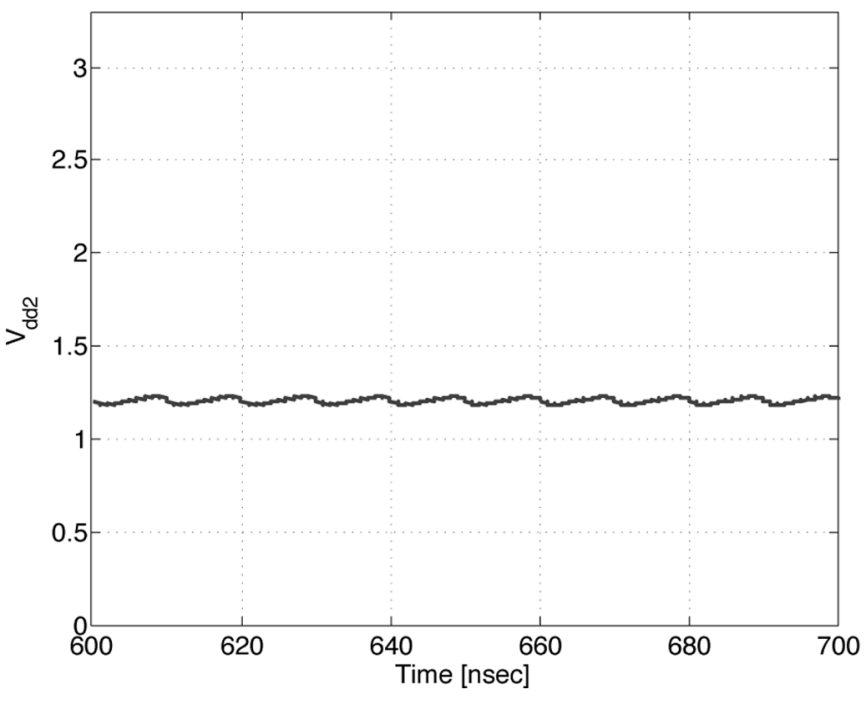

Fig. 9. DC voltage at the output of a distributed filter.

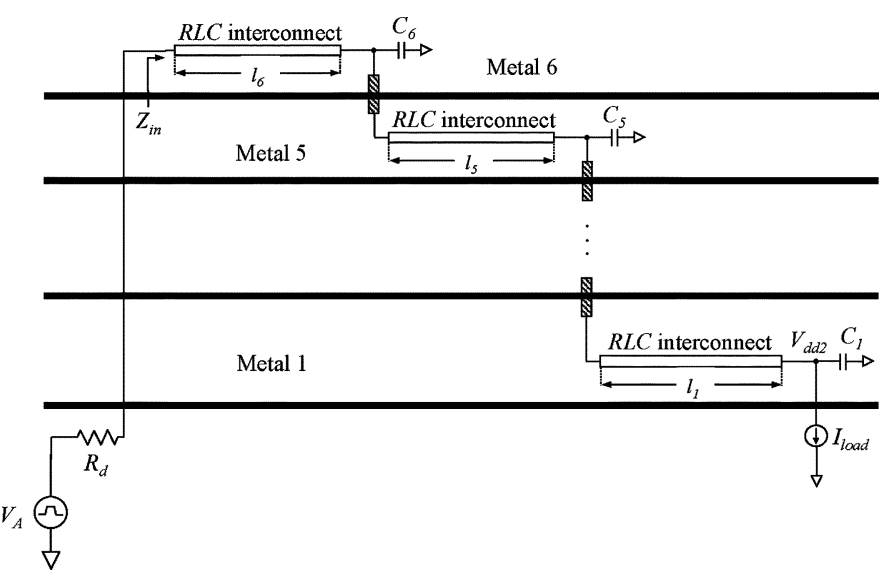

Fig. 10. Distributed filter in a 2-D technology.

lengths and capacitances are therefore chosen, producing a 1.2 volt power supply with $700 \mathrm{~mA}$ maximum current, resulting in a total of $4.2 \mathrm{nF} / \mathrm{plane}$. The interconnect network and the capacitors per plane occupy, in this example, about $0.42 \mathrm{~mm}^{2}$.

\section{Distributed Filter in a 2-D Technology}

As mentioned in Section II, the distributed filter can also be implemented in a 2-D technology, as shown in Fig. 10. In a 2-D technology, the filter spans all metal layers down to the device layer where conventional vias are used to connect the different metal layers.

To provide an effective comparison to the 3-D case, a 180 nm 2-D TSMC technology with six copper metal layers is used. Additionally, the width of all of the metal interconnects and the output resistance of the driver are assumed to be $4 \mu \mathrm{m}$ and $R_{d}=$ $7.36 \Omega$, respectively, as in the 3-D case. The $R L C$ parameters for this technology are listed in Table II.

Following the same design methodology and performance requirements as used for the 3-D case, the magnitude of the transfer function at $\omega_{s}$ for different capacitances and interconnect lengths, assuming $l=l_{1}=l_{2}=l_{3}=l_{4}=l_{5}=l_{6}$ and $C=C_{1}=C_{2}=C_{3}=C_{4}=C_{5}=C_{6}$, is depicted in 
TABLE II

$R L C$ INTERCONNECT (COPPER) IMPEDANCES IN A 2-D TECHNOLOGY

\begin{tabular}{cccc}
\hline & $R[m \Omega / \mu \mathrm{m}]$ & $L[\mathrm{pH} / \mu \mathrm{m}]$ & $C[\mathrm{fF} / \mu \mathrm{m}]$ \\
\hline \hline $\mathbf{M}_{6}$ & 5.5 & 1.3 & 1.0 \\
\hline $\mathrm{M}_{5}, \mathrm{M}_{4}, \mathrm{M}_{3}, \mathrm{M}_{2}, \mathrm{M}_{1}$ & 10.40 & 1.30 & 0.89 \\
\hline
\end{tabular}

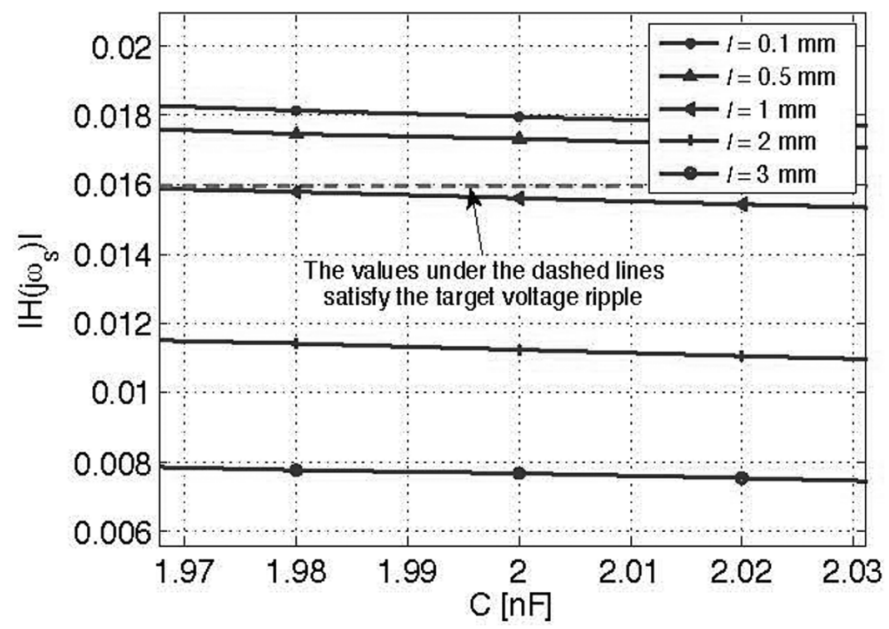

Fig. 11. Magnitude of the transfer function at $\omega_{s}$ for different line lengths and capacitances in a 2-D technology.

TABLE III

PERFormance COMPARISON OF THE DistRIBUTEd FILTER IMPLEMENTEd IN A 2-D AND 3-D TECHNOLOGY

\begin{tabular}{ccc}
\hline & 2-D $180 \mathrm{~nm}$ & 3-D $180 \mathrm{~nm}$ \\
\hline \hline Capacitance density $\left[\mathrm{fF} / \mu \mathrm{m}^{2}\right]$ & 1 & 10 \\
\hline Total capacitance $[\mathrm{nF}]$ & 12 & 4.2 (per plane) \\
\hline filter efficiency $[\%]$ & 88 & 88 \\
\hline Area $\left[\mathrm{mm}^{2}\right]$ & 12.25 & 0.42 \\
\hline
\end{tabular}

Fig. 11. To ensure a proper comparison with the 3-D case, the interconnect length in this example is also $l=1 \mathrm{~mm}$, resulting in a capacitance $C=2 \mathrm{nF}$ (see Fig. 11). The total on-chip capacitance in the $2-\mathrm{D}$ case is therefore $2 \times 6=12 \mathrm{nF}$. The required duty cycle of the signal driving the power MOSFETs is $D_{\mathrm{PWM}}=0.73$, and the efficiency of the filter is about $88 \%$. The area occupied by this filter is about $12.25 \mathrm{~mm}^{2}$.

A comparison of the filter implemented in a 2-D and 3-D technology is provided in Table III. Note that the 3-D implementation exhibits better characteristics due to several reasons. The on-chip capacitance density is ten times larger in a 3-D technology, translating into a larger area occupied by the filter in a 2-D technology. Additionally, in the 3-D technology, the capacitance is distributed among the three planes. Furthermore, the capacitance required in the 2-D technology is 2.85 larger than the capacitance in the 3-D case.

The requirement for on-chip capacitance in the 2-D technology increases due to the following rationale. The cutoff frequency of the distributed low pass filter is proportional to $1 / 2 \pi R_{\text {int }} C_{\text {plane }}$, where $R_{\text {int }}$ is the total resistance of the filter interconnects and $C_{\text {plane }}$ is the total on-chip capacitance connected to the interconnects on different planes, $C_{\text {plane }}=\sum_{i=1}^{n} C_{i}$ (see Fig. 4). To maintain the same cutoff frequency as in the 3-D case and since $R_{\text {int }}$ is lower in the 2-D case, the requirement for $C_{\text {plane }}$ increases (in this example, by $2.85)$. The resistance $R_{\text {int }}$ in the 2-D case is lower as compared to the 3-D technology since in the 2-D technology, copper interconnects are used while aluminum interconnects are used in the 3-D technology.

Finally, the efficiency of the filter in both cases is approximately the same (88\%). Summarizing, the distributed filter requires significantly less total area when implemented within a 3-D technology as compared to a 2-D technology (in this example, 30 times less, as inferred from Table III).

\section{Conventional Filter}

A conventional buck converter filter is composed of a second order low pass $L C$ filter, as shown in Fig. 2(a). Assuming the inductor and capacitor exhibit ideal characteristics, the transfer function of the $L C$ filter is

$$
H_{\text {rect }, L C}(j \omega)=\frac{1}{1-\omega^{2} L C+j \omega C R_{d}}
$$

where $R_{d}, L$, and $C$ are the effective output resistance of the power MOSFETs and the inductor and capacitor of the conventional filter, respectively.

In this example, a practical $2 \mathrm{nH}$ on-chip inductor is used, based on the characterization in [17]. This inductor has been designed in a 130-nm CMOS technology, conducting $130 \mathrm{~mA}$ maximum current, with $75 \mu \mathrm{m}$ line width and $600 \mu \mathrm{m}$ diameter. In order to conduct $700 \mathrm{~mA}$, five $2 \mathrm{nH}$ inductors are connected in parallel, resulting in $0.4 \mathrm{pH}$ total inductance. The dc-dc conversion is from 3.3 to $1.2 \mathrm{~V}(D=0.36)$ at a switching frequency $f_{s}=100 \mathrm{MHz}$. The effective output resistance of the power MOSFETs in a $0.18-\mu \mathrm{m}$ CMOS technology delivering $700 \mathrm{~mA}$ is $R_{d}=7.36 \Omega$. To achieve $5 \%\left(V_{r}=0.05 V_{\mathrm{dd} 2}\right.$ peak-to-peak) ripple voltage, the magnitude of the transfer function at $100 \mathrm{MHz}$ according to (13) is 0.016 . To satisfy these requirements, the corner frequency of an $L C$ low pass filter, i.e., the resonant frequency, should be about one decade less than $f_{s}$.

This condition results in $C=20 \mathrm{nF}$ and five $L=0.4 \mathrm{pH}$ inductors. Note that for these performance requirements, significant area is required. The inductors and capacitor occupy about $4 \mathrm{~mm}^{2}$ as compared to $0.42 \mathrm{~mm}^{2}$ per plane occupied by the distributed filter.

\section{Performance Analysis}

To obtain deeper insight into the performance characteristics of the proposed filter, an analysis over a wide range of design parameters is presented in this section. In all cases, the filter is designed to satisfy less than $5 \%$ voltage ripple with an input $3.3 \mathrm{dc}$ voltage. In the simulation of a 3-D filter, the parameters and structure presented in the case study discussed in Section IV are assumed.

To place these results in the context of a conventional filter, the inductor and capacitor values as a function of switching frequency for different current loads are illustrated in Fig. 12. As 


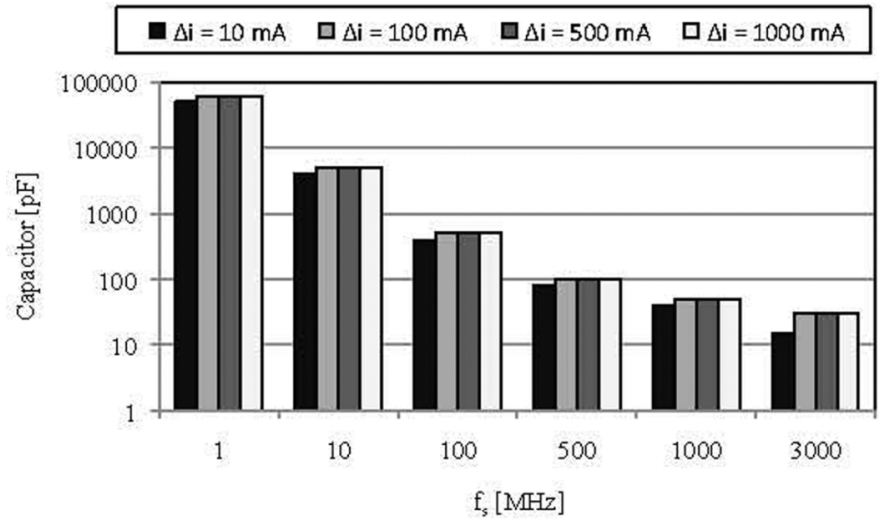

(a)

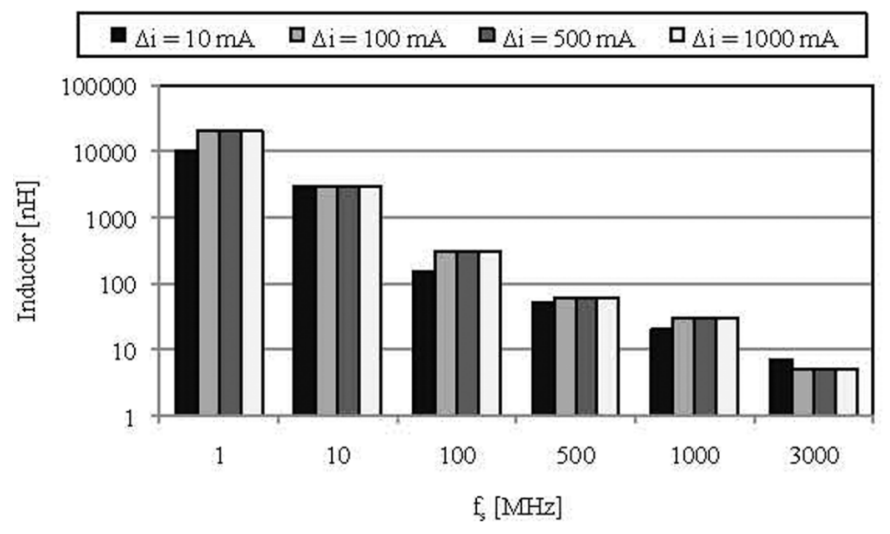

(b)

Fig. 12. Conventional $L C$ filter: (a) capacitance and (b) inductance.

expected, the magnitude of the lumped inductor and capacitor decreases as the switching frequency increases. However, in the megahertz frequency region, the magnitude of the capacitor is between $100 \mathrm{pF}$ and $100 \mathrm{nF}$, while the magnitude of the inductor is between $60 \mathrm{nH}$ and $10 \mu \mathrm{H}$. In current technologies, these large inductors cannot be implemented on-chip. When the switching frequency is increased to gigahertz frequencies, the magnitude of the on-chip inductor becomes realizable on-chip. Note that in this case, the current load is assumed to only affect the output resistance of the power MOSFETs. Therefore, at higher current loads (above $100 \mathrm{~mA}$ ), insignificant changes in the magnitude of the capacitance and inductance can be observed in Fig. 12.

The efficiency, total capacitance, and area per plane of the distributed filter as a function of switching frequency for different current loads are shown in Fig. 13. At a $1 \mathrm{MHz}$ switching frequency, the efficiency of the filter reaches $80 \%$. At higher frequencies, the efficiency is significantly greater, reaching $98 \%$ at $500 \mathrm{MHz}, 1 \mathrm{GHz}$, and $3 \mathrm{GHz}$. Since only the filter efficiency is considered, excluding the power MOSFETs, the efficiency at the higher frequencies is dependent on the input impedance of the distributed filter which does not change significantly at higher frequencies. The input impedance remains approximately the same at higher frequencies since the capacitors $C_{1}$ to $C_{n}$ of the distributed filter contribute less to the input impedance. As expected, the total capacitance and area occupied by the distributed filter decreases as the frequency increases. This be-

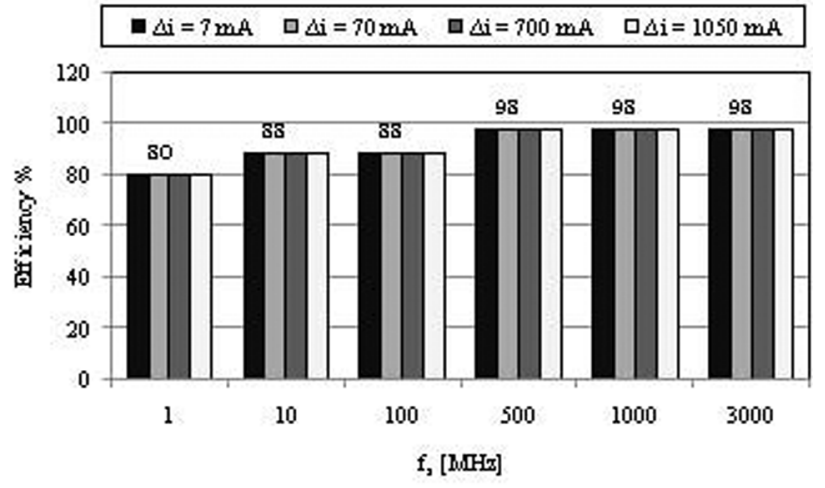

(a)

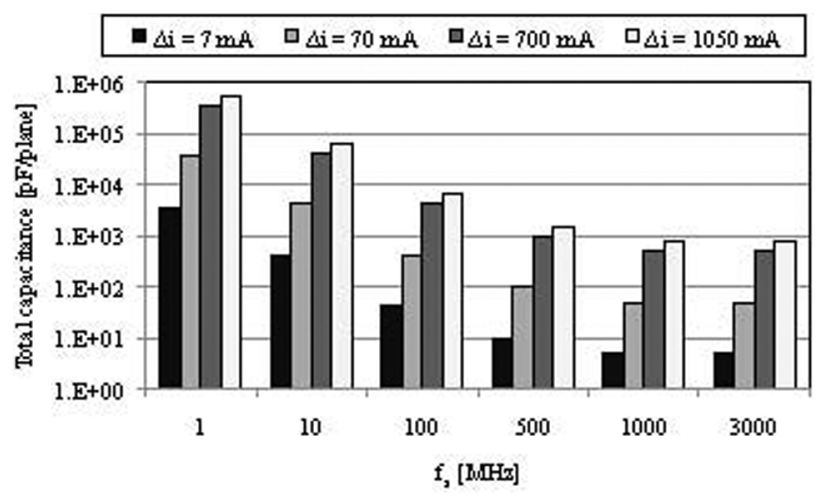

(b)

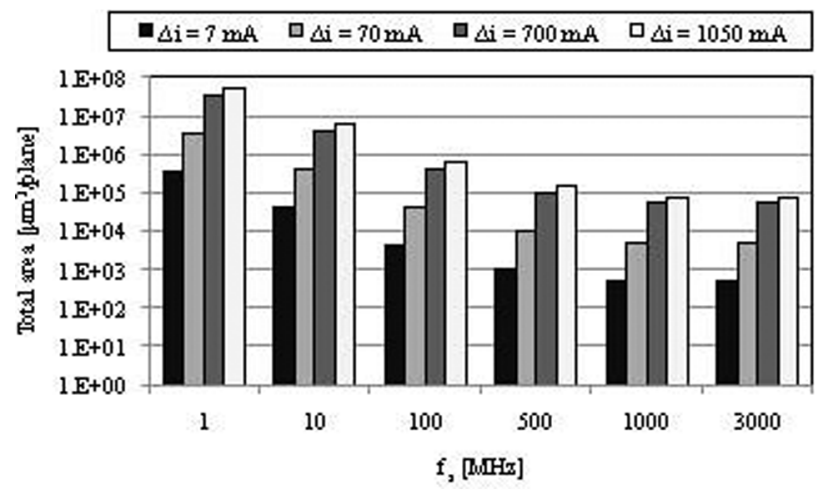

(c)

Fig. 13. 3-D distributed filter: (a) efficiency, (b) total capacitance per plane, and (c) total area per plane.

havior occurs since less capacitance and shorter interconnects are required at higher frequencies to satisfy the target voltage ripple.

As shown in Fig. 12(b), the magnitude of the required inductor within an $L C$ filter varies between 50 and $10000 \mathrm{nH}$ for switching frequencies up to $1 \mathrm{GHz}$. The large range of inductance is attributed to the wide range of switching frequencies, i.e., $1 \mathrm{MHz}$ to $1 \mathrm{GHz}$. At $3 \mathrm{GHz}$, the required inductor is less than $10 \mathrm{nH}$. This characteristic implies that at switching frequencies used in current technologies, i.e., up to several megahertz [26], a conventional $L C$ filter cannot be implemented on-chip.

To evaluate the capability of providing a wide voltage conversion range, as required in 3-D circuits, the proposed methodology is compared to three on-chip converters [17], [27], [28], as shown in Fig. 14. The proposed converter in this example 


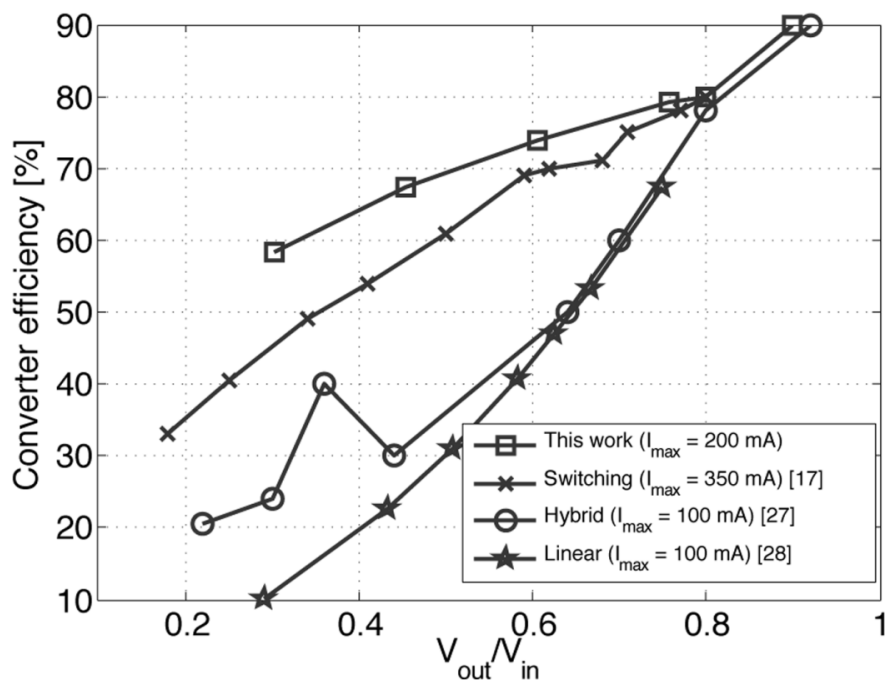

Fig. 14. Comparison of power efficiency.

distributes $200 \mathrm{~mA}$ maximum current. As evident from Fig. 14, the power efficiency of the proposed converter is greater than the other converters. A primary reason for the superior power efficiency is that the proposed converter eliminates the need for an on-chip inductor and any associated losses.

\section{CONCLUSION}

Integrating dc-dc converters on-chip can significantly enhance the performance and reliability of integrated circuits. Conventional filters, however, are difficult to integrate due to the large magnitude of the on-chip inductors and capacitors. A distributed buck converter filter for application to three-dimensional circuits is proposed in this paper. By exploiting the low pass bandwidth properties of transmission lines, simultaneously generating and distributing power supplies to different planes is possible. An example converter based on the MITLL 3-D CMOS technology is described, demonstrating a dc-dc converter that can generate a $1.2 \mathrm{~V}$ power supply while delivering $700 \mathrm{~mA}$.

A performance analysis considering the filter efficiency, total capacitance, and physical area is performed for a wide range of switching frequencies and current loads. A conventional on-chip $L C$ filter in the megahertz frequency range is not practical due to the large inductors. Alternatively, an on-chip distributed filter can be implemented in the megahertz frequency range, achieving high efficiency, suitable for 3-D integration.

\section{REFERENCES}

[1] V. F. Pavlidis and E. G. Friedman, Three-Dimensional Integrated Circuit Design. San Mateo, CA: Morgan Kaufmann, 2009.

[2] J. Sun, D. Giuliano, S. Devarajan, J.-Q. Lu, T. P. Chow, and R. J. Gutmann, "Fully monolithic cellular buck converter design for 3-D power delivery," IEEE Trans. Very Large Scale Integr. (VLSI) Syst., vol. 17, no. 3, pp. 447-451, Mar. 2009.

[3] R. G. Gutmann, J. Q. Lu, Y. Kwon, J. F. McDonald, and T. S. Cale, "Three-dimensional ICs: A technology platform for integrated systems and opportunities for new polymeric adhesives," in Proc. IEEE Int. Conf. Polymers Adhesives Microelectron. Photon., Oct. 2001, pp. 173-180.
[4] C. S. Tan, R. J. Gutmann, and L. R. Reif, Wafer Level 3-D ICs Process Technology. New York: Springer, 2008.

[5] V. Kursun and E. G. Friedman, Multi-Voltage CMOS Circuit Design. New York: Wiley, 2006.

[6] R. W. Erickson and D. Maksimovic, Fundamentals of Power Electronics. Norwell, MA: Kluwer, 2001.

[7] J. Rosenfeld and E. G. Friedman, "On-chip DC-DC converters for three-dimensional ICs," in Proc. IEEE Int. Symp. Quality Electron. Des., Mar. 2009, pp. 759-764.

[8] H. Kim, H.-J. Kim, and W.-S. Chung, "Pulsewidth modulation circuits using CMOS OTAs," IEEE Trans. Circuits Syst. I, Reg. Papers, vol. 54, no. 9, pp. 1869-1878, Sep. 2007.

[9] B. Sahu and G. A. Rincon, "An accurate, low-voltage, CMOS switching power supply with adaptive on-time pulse-frequency modulation (PFM) control," IEEE Trans. Circuits Syst. I, Reg. Papers, vol. 54, no. 2, pp. 312-321, Feb. 2007.

[10] B. S. Cherkauer and E. G. Friedman, "A unified design methodology for CMOS tapered buffers," IEEE Trans. Very Large Scale Integr. (VLSI) Syst., vol. 3, no. 1, pp. 99-111, Mar. 1995.

[11] R. S. Kagan and M. Chi, "Improving power supply efficiency with mosfet synchronous rectifiers," in Proc. IEEE Int. Conf. Polymers Adhesives Microelectron. Photon., Jul. 1982, pp. D4.1-D4.9.

[12] V. Kursun, S. G. Narendra, V. K. De, and E. G. Friedman, "Lowvoltage-swing monolithic DC-DC conversion," IEEE Trans. Circuits Syst. II, Exp. Briefs, vol. 51, no. 5, pp. 241-248, May 2004.

[13] V. Kursun, S. G. Narendra, V. K. De, and E. G. Friedman, "Cascode monolithic DC-DC converters for reliable operation at high input voltages," Analog Integr. Circuits Signal Process., vol. 42, no. 3, pp. 231-238, May 2005.

[14] V. Kursun, V. K. De, E. G. Friedman, and S. G. Narendra, "Monolithic voltage conversion in low-voltage CMOS technologies," Microelectron. J., vol. 36, no. 9, pp. 863-867, May 2005.

[15] A. M. Niknejad and R. G. Meyer, "Analysis design, and optimization of spiral inductors and transformers for Si RF ICs," IEEE J. Solid-State Circuits, vol. 33, no. 10, pp. 1470-1481, Oct. 1998.

[16] W.-Z. Chen, W.-H. Chen, and K.-C. Hsu, "Three-dimensional fully symmetric inductors, transformer, and Balun in CMOS technology," IEEE Trans. Circuits Syst. I, Reg. Papers, vol. 54, no. 7, pp. 1413-1423, Jul. 2007.

[17] J. Wibben and R. Harjani, "A high-efficiency DC-DC converter using 2 nH integrated inductors," IEEE J. Solid-State Circuits, vol. 43, no. 4, pp. 844-854, Apr. 2008.

[18] T. Karnik et al., "High-frequency DC-DC conversion: Fact or fiction," in Proc. IEEE Int. Symp. Circuits Syst., May 2006, pp. 245-248.

[19] T. Suetsugu and M. K. Kazimierczuk, "Integration of class DE inverter for on-chip DC-DC power supplies," in Proc. IEEE Int. Symp. Circuits Syst., May 2006, pp. 3133-3136.

[20] P. Hazucha, G. Schrom, H. Jaehong, B. A. Bloechel, P. Hack, G. E. Dermer, S. Narendra, D. Gardner, T. Karnik, V. De, and S. Borkar, “ A $233-\mathrm{MHz} 80 \%-87 \%$ efficient four-phase DC-DC converter utilizing air-core inductors on package," IEEE J. Solid-State Circuits, vol. 40, no. 4, pp. 838-845, Apr. 2005.

[21] MIT Lincoln Laboratories, Cambridge, "MITLL low-power FDSOI CMOS process application notes," 2006.

[22] MIT Lincoln Laboratories, Cambridge, "MITLL low-power FDSOI CMOS process design guide," 2006.

[23] Nanoscale Integration and Modeling Group, Arizona State Univ., Tempe, "Predictive technology model," [Online]. Available: http://www.eas.asu.edu/ ptm

[24] I. Savidis and E. G. Friedman, "Electrical characterization and modeling of 3-D vias," in Proc. IEEE Int. Symp. Circuits Syst., May 2008, pp. 784-787.

[25] I. Savidis and E. G. Friedman, "Closed-form expressions of 3-D via resistance, inductance, and capacitance," IEEE Trans. Electron Devices, vol. 56, no. 9, pp. 1873-1881, Sep. 2009.

[26] R. W. Erickson and D. Maksimovic, Fundamentals of Power Electronics. New York: Springer, 2004.

[27] G. Patounakis, Y. W. Li, and K. L. Shepard, "A fully integrated on-chip DC-DC conversion and power management system," IEEE J. SolidState Circuits, vol. 39, no. 3, pp. 443-452, Mar. 2004.

[28] P. Hazucha, T. Karnik, B. A. Bloechel, C. Parsons, D. Finan, and S. Borkar, "Area-efficient linear regulator with ultra-fast load regulation," IEEE J. Solid-State Circuits, vol. 40, no. 4, pp. 933-940, Apr. 2005. 


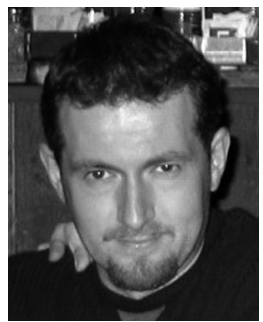

Jonathan Rosenfeld received the B.S. degree in mechanical engineering from Technion-Israel Institute of Technology, Haifa, Israel, in 1999, the Bachelors of Technology degree (with honors) in electronic engineering from Ort-Braude College, Karmiel, Israel, in 2003, and the M.S. and Ph.D. degrees in electrical and computer engineering from the University of Rochester, Rochester, NY, in 2005 and 2009, respectfully.

He was an intern with Intrinsix Corporation, Fairport, NY, in 2005, where he designed Gm-C circuits for $\Sigma \Delta$-modulator ADC for FM tuner. In 2007, he was an intern with Eastman Kodak Company, Rochester, NY, where he developed column-based multiple ramp integrated $\mathrm{ADC}$ for CMOS image sensors. His research interests include the areas of interconnect design, resonant clock and data distribution networks, on-chip DC-DC converters, 3-D circuits, and the design of analog and mixedsignal integrated circuits.

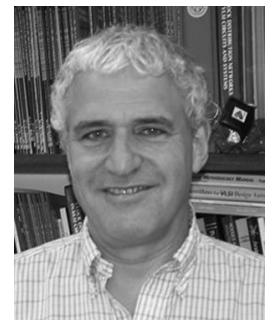

Eby G. Friedman (F'00) received the B.S. degree from Lafayette College, Easton, PA, in 1979, and the M.S. and Ph.D. degrees from the University of California, Irvine, in 1981 and 1989, respectively, all in electrical engineering.

From 1979 to 1991, he was with Hughes Aircraft Company, rising to the position of manager of the Signal Processing Design and Test Department, responsible for the design and test of high performance digital and analog IC's. He has been with the Department of Electrical and Computer Engineering, University of Rochester, Rochester, NY, since 1991, where he is a Distinguished Professor. He is also a Visiting Professor at the Technion-Israel Institute of Technology, Haifa, Israel. His current research and teaching interests include high performance synchronous digital and mixed-signal microelectronic design and analysis with application to high speed portable processors and low power wireless communications. He is the author of almost 400 papers and book chapters, several patents, and the author or editor of 13 books in the fields of high speed and low power CMOS design techniques, high speed interconnect, and the theory and application of synchronous clock and power distribution networks.

Dr. Friedman is the Regional Editor of the Journal of Circuits, Systems and Computers, Chair of the IEEE TRANSACTIONS ON VERY LARGE SCALE INTEGRATION (VLSI) SYSTEMS steering committee, and a Member of several editorial boards and conference technical program committees. He previously was the Editor-in-Chief of the IEEE TRANSACTIONS ON VERY LARGE SCALE INTEGRATION (VLSI) SYSTEMS, a Member of the editorial board of the PROCEEDINGS OF THE IEEE, a Member of the Circuits and Systems (CAS) Society Board of Governors, Program and Technical chair of several IEEE conferences. He was a recipient of the University of Rochester Graduate Teaching Award, and the College of Engineering Teaching Excellence Award. He is a Senior Fulbright Fellow. 